\title{
Association of Residential Proximity to the Coast With Incident Myocardial Infarction: A Prospective Cohort Study
}

\section{OPEN ACCESS}

Edited by:

Stéphane Cook,

Université de Fribourg, Switzerland

Reviewed by:

Xin Li,

Third Affiliated Hospital of Sun Yat-sen

University, China

Jiezhen Qi,

Dongguan University of

Technology, China

*Correspondence:

Liao Xin-xue

liaoxinx@mail.sysu.edu.cn

Liao Li-zhen

liaolizhen2013gy@163.com

†These authors have contributed equally to this work

Specialty section:

This article was submitted to

Coronary Artery Disease,

a section of the journal

Frontiers in Cardiovascular Medicine

Received: 04 August 2021 Accepted: 10 January 2022

Published: 17 February 2022

Citation:

Xiao-dong Z, Shao-zhao Z, Xun H,

Xin-xue $L$ and Li-zhen L (2022)

Association of Residential Proximity to

the Coast With Incident Myocardial Infarction: A Prospective Cohort

Study.

Front. Cardiovasc. Med. 9:752964. doi: 10.3389/fcrm.2022.752964

\begin{abstract}
Zhuang Xiao-dong ${ }^{1,2+}$, Zhang Shao-zhao ${ }^{1,2+}$, Hu Xun ${ }^{1,2+}$, Liao Xin-Xue ${ }^{1,2 *}$ and Liao Li-zhen ${ }^{3,4 *}$

${ }^{1}$ Department of Cardiology, The First Affiliated Hospital of Sun Yat-sen University, Guangzhou, China, ${ }^{2}$ NHC Key Laboratory of Assisted Circulation, Sun Yat-sen University, Guangzhou, China, ${ }^{3}$ Health Department, Guangdong Provincial Key Laboratory of Pharmaceutical Bioactive Substances, Guangzhou Higher Education Mega Center, Guangdong Pharmaceutical University, Guangzhou, China, ${ }^{4}$ Guangdong Engineering Research Center for Light and Health, Guangzhou

Higher Education Mega Center, Guangzhou, China
\end{abstract}

Background: Little is known about how the residential distance to the coast is associated with incident myocardial infarction (MI) and which mechanisms may explain the association. We aim to explore this association using data from a prospective, population-based cohort with unprecedented sample size, and broad geographical coverage.

Methods: In this study, 377,340 participants from the UK Biobank were included.

Results: It was shown that 4,059 Ml occurred during a median 8.0 years follow-up. Using group $(<1 \mathrm{~km})$ as reference, group $(20-50 \mathrm{~km})$ was associated with a lower risk of $\mathrm{Ml}$ (hazard ratio, $\mathrm{HR} 0.79,95 \% \mathrm{Cl}$ 0.64-0.98) and a U-shaped relation between distance to the coast and Ml was shown with the low-risk interval between 32 and $64 \mathrm{~km}$ ( $\left.p_{\text {non-linear }}=0.0012\right)$. Using participants of the intermediate region $(32-64 \mathrm{~km})$ as a reference, participants of the offshore region $(<32 \mathrm{~km})$ and inland region $(>64 \mathrm{~km})$ were both associated with a higher risk of incident $\mathrm{Ml}(H R$ 1.12, 95\% Cl 1.04-1.21 and $H R$ 1.09, 95\% Cl 1.01-1.18, respectively). HR for offshore region ( $<32 \mathrm{~km})$ was larger in subgroup with low total physical activity (<24 h/week) (HR 1.24, 95\% Cl 1.09-1.42, $\left.p_{\text {interaction }}=0.043\right)$. HR for inland region $(>64 \mathrm{~km})$ was larger in subgroup in urban area $\left(H R 1.12,95 \% \mathrm{Cl} 1.03-1.22, p_{\text {interaction }}=0.065\right)$ and in subgroup of high nitrogen dioxide $\left(\mathrm{NO}_{2}\right)$ air pollution $\left(H R 1.29,95 \% \mathrm{Cl} 1.11-1.50, p_{\text {interaction }}=0.021\right)$.

Conclusion: We found a $U$-shaped association between residential distance to the coast and incident MI, and the association was modified by physical activity, population density, and air pollution.

Keywords: myocardial infarction, distance to coast, cohort, UK Biobank, association

\section{KEYPOINTS}

Question

- Proximity to the coast, an essential natural outdoor environment attribute, is positively related to self-reported general and mental health. 


\section{Findings}

- We found a $U$-shaped association between residential distance to the coast and incident myocardial infarction (MI). The association of offshore region with incident MI was modified by total physical activity. The association of inland region with incident MI was modified by urban/rural area or nitrogen dioxide $\left(\mathrm{NO}_{2}\right)$ air pollution.

Meaning

- The harmful effect of residential distance to coast on incident MI may vary with the distance of coastline and is regulated by various factors. Therefore, when possible, advice on the living environment and health should be personalized.

\section{BACKGROUND}

Natural outdoor environment attributes, such as green spaces (i.e., forests or parks), blue spaces (i.e., visible bodies of water), and coastal proximity, have long-term effects on behavior and health (1-3). Researchers have provided preliminary evidence that proximity to the coast, an essential natural outdoor environment attribute, is positively related to both self-reported general and mental health, and the beneficial effect of coastal proximity was mainly mediated by improving behavioral pathways (such as physical activity, sleep, and diet), alleviating stress, and avoiding environmental pollution (4-6). However, most of these studies are limited by a small sample size, weak geographical representation, insufficient adjustment for confounders, and unclear definition of exposure. Besides, most of the outcomes of previous reports were self-reported and not relating to specific diseases. Hence, little is known about how the residential distance to the coast is associated with the incidence of myocardial infarction (MI) and which mechanisms may explain the association.

To deal with these limitations, we explored the association between distance to the coast and incident MI using data from UK Biobank, a prospective, population-based cohort study with unprecedented sample size and broad geographical coverage.

\section{METHODS}

\section{Study Population}

UK Biobank is a large prospective cohort of middle-aged adults designed to support biomedical analysis focused on improving the prevention, diagnosis, and treatment of chronic disease, the methods and aim of which have been reported elsewhere (7). In brief, between April 2007 and December 2010, UK Biobank recruited 502,628 participants (5.5\% response rate, most of whom were age 40-70 years) from the general population (8). Participants attended 1 of 22 assessment centers across England, Wales, and Scotland and completed a touch screen questionnaire, had physical measurements taken, and provided biological samples. All participants provided written informed

Abbreviations: $\mathrm{MI}$, myocardial infarction; $\mathrm{NO}_{2}$, nitrogen dioxide; $\mathrm{PM}$, particulate matter; HR, hazard ratios; Q1, first quintile; CVDs, cardiovascular diseases. consent, and the study was approved by the NHS National Research Ethics Service. This research has been conducted using the UK Biobank Resource under Application Number 56,925.

In the present study, we included participants with data of distance from participant's residence location to the coast $(n=440,874)$, excluded participants with previous cardiovascular diseases (CVDs) (coronary heart disease and stroke, $n=28,980)$ or cancer $(n=34,544)$ at baseline, leaving 377,340 participants remained for analysis (Supplementary Figure S1).

\section{Ascertainment of Outcome}

In UK Biobank, hospital admissions were identified via record linkage to Health Episode Statistics records for England and Wales and the Scottish Mortality Records for Scotland (8). Detailed information about recorded linkage procedures is available online. Incident MI, comprising fatal and non-fatal ST-segment elevation and non-ST-segment elevation MI, was defined as ICD 10 (international classification of diseases, 10th revision) code of I21, I21.4, and I21.9 recorded on hospital admission. At the time of analysis, the last recorded MI was on March 31, 2017, which was used as the censoring date for other participants if no outcome had been recorded, whichever occurred first.

\section{Ascertainment of Exposures}

Environmental indicators attributed to participants were based on home location grid references. Data on the natural environment were linked using CEH 2007 Land Cover Map data. Measures of residential greenspace were estimated for England residents using the 2005 Generalized Land Use Database for England. It provides data on land use distribution for 2001 Census Output Areas in England and is consistent with the previous related research $(9,10)$. Residential distance to the coast was defined as the participant's residence location to the coast according to the participant's address, measured in Kilometers $(\mathrm{km})$. The Euclidean distance raster from the coastline was calculated for a small grid cell size, then values from the grid allocated to UKB point locations. Based on existing literature, distances to the coast were collapsed into five categories: $0-1 \mathrm{~km}$, $1-5 \mathrm{~km}, 5-20 \mathrm{~km}, 20-50 \mathrm{~km}$, and over $50 \mathrm{~km}$ (11). To obtain approximately equal sample sizes per category, we divided the data into five quintiles for the current analyzes.

\section{Data on Potential Confounders and Effect Modifiers}

Sociodemographic factors (age, gender, ethnicity, Townsend deprivation index, professional qualifications, income, employment, and month of recruitment), health-related variables (overall health rating, mental health, handgrip strength, family history of heart diseases, medication for aspirin, cholesterol, and blood pressure, prevalent diabetes, and hypertension at baseline), lifestyle factors (smoking status, drinking status, body mass index, total physical activity, sedentary lifestyle, sleep duration, and dietary intake), residential air and noise pollution (nitrogen oxides, nitrogen dioxide $\left[\mathrm{NO}_{2}\right]$, particulate matter $[\mathrm{PM}]$, traffic intensity, average daytime/night sound level of noise pollution), 
home area population density classified as urban or rural, and greenspace (domestic garden percentage, greenspace percentage, natural environment percentage, and water percentage) were treated as potential confounders.

Age was calculated from dates of birth and baseline assessment. Qualification, average total household income, current employment status, overall health rating, mental health status, family history of heart diseases, the medication used, and sleep pattern were recorded using an electronic questionnaire completed by participants. Smoking status and drinking status were categorized into never, former and current smoker or drinker. Area-based socioeconomic status was derived from the postal code of residence by using the Townsend deprivation score (12). Dietary information was collected via the Oxford WebQ; a web-based 24 recall questionnaire developed specifically for large population studies (13). Physical activity was based on self-report by using the International Physical Activity Questionnaire short form, and total physical activity was calculated as the sum of walking, moderate, and vigorous exercise measured as metabolic equivalents (MET-h/week) (14). Grip strength was accessed through the use of a hydraulic hand dynamometer while sitting $(14,15)$. Total time spent in sedentary behaviors was derived from the sum of self-reported time spent driving, using a computer, and watching television. Land use regression (LUR)-based estimates of $\mathrm{NO}_{2}, \mathrm{PM} 10$, and PM2.5 for 2010 were generated as part of the European Study of Cohorts for Air Pollution Effects (ESCAPE) and link to geocoded residential addresses of UK Biobank participants (16). Noise estimates were derived from a simplified version of the Common Noise Assessment Methods in the European Union (CNOSSOS-EU) framework (17). Home area population density classified as urban or rural was derived by combining each participant's home postcode with data generated from the 2001 census from the Office of National Statistics, using the Geoconvert tool from Census Dissemination Unit. More details for each variable are available on the UK Biobank website http://www.ukbiobank.ac.uk/.

\section{Statistical Analysis}

Baseline characteristics of 377,340 participants were described as means or percentages and were compared between groups using the one-way ANOVA test, the $\chi 2$ test, and the Kruskal-Wallis test, as appropriate. We coded missing data as a missing indicator category for categorical variables, such as smoking status, and mean values for continuous variables.

The association between residential distance to coast and MI was explored using Cox proportional hazard models. The proportional hazard assumption was checked by tests based on Schoenfeld residuals. The results were reported as hazard ratios (HRs) together with 95\% CIs. First, distance to the coast was treated as continuous variables, and HRs were calculated per $1 \mathrm{SD}(26.7 \mathrm{~km})$ difference in distance to the coast. Then we categorized the distance to coast into $<1 \mathrm{~km}, 1-5 \mathrm{~km}, 5-20 \mathrm{~km}$, $20-50 \mathrm{~km}$, and $\geq 50 \mathrm{~km}$ groups and calculated the HRs for the other four groups taking the first group $(<1 \mathrm{~km})$ as reference. We also categorized distance to coast into quintiles (Q1-Q5) base on the sample distribution and calculated the HRs for the last four groups taking the first quintile (Q1) as reference. Models were arranged a priori to investigate the impact of incremental adjustment. Model 1 adjusted for age, gender, ethnicity, social deprivation, income, employment status, total physical activity, overall health rating, smoking, drinking status, BMI, and handgrip strength. Model 2 additionally adjusted for family history of heart diseases, medication for aspirin, cholesterol, and blood pressure, prevalent diabetes, and hypertension. Model 3 further adjusted for air pollution, noise pollution, sleep duration, dietary intake, and home area population density.

To examine the overall statistical significance and the non-linearity of the exposure, we used likelihood ratio tests. A multivariable restricted cubic spline with 3 knots was used to express the dose-response relationship. We calculated $H R s$ for living in the offshore region $(<32 \mathrm{~km})$ and inland region $(>64 \mathrm{~km})$ using Cox-proportional hazard models with incremental adjustment separately, taking participants in the intermediate area $(32-64 \mathrm{~km})$ within the lowest risk interval as a reference, according to the result of the restricted cubic spline. We conducted subgroup analyses to assess potential modification effects by the following factors: sex, age, BMI, sedentary behavior, sleep duration, total physical activity, smoking status, drinking status, income, area-based socioeconomic status, mental health status, urban area, air pollution, noise pollution, and hypertension. A sensitivity analysis was also conducted to investigate the effect of removing MI occurring within the first 24 months of follow-up to reduce the possible impact of reverse causation. Effect modifiers were investigated by adding to the fully adjusted model an interaction term between exposure and each of these variables.

All analyses were performed with SPSS V26 (IBM) and Stata V15 (Stata Corporation, College Station, TX, USA). A two-sided value of $p<0.05$ was considered statistically significant.

\section{RESULTS}

The baseline characteristics of the participants are summarized in Table 1. The mean value of residential distance to the coast for 377,340 participants was $45.7 \pm 26.7 \mathrm{~km}$. Briefly, participants who lived proximal to the coast $(<1 \mathrm{~km})$ were more likely to be white, retired, less social deprivation, less diabetes, more physically active, and exposed to less $\mathrm{NO}_{2}$ air pollution and traffic intensity. Supplementary Table $\mathbf{S 1}$ shows the characteristics of participants by quintiles (Q1-Q5) of residential distance to the coast.

During a median of 8.0 years (3.0 million person-years) of follow-up, 4,059 cases of MI occurred. Table 2 shows the association between residential distance to the coast and incident MI. After adjusting potential confounders, no significant association with MI was observed for distance to coast as continuing variable (HR 0.98 per SD increase, 95\% CI 0.95$1.02, p=0.321)$. Using group $(<1 \mathrm{~km})$ as reference, group (20$50 \mathrm{~km}$ ) was associated with a statistically significant lower risk of MI (HR 0.79, 95\% CI 0.64-0.98, $p=0.033$ ). When using the lowest quintile Q1 $(<14.1 \mathrm{~km})$ as reference, Q2 $(14.1-40.2 \mathrm{~km})$, and Q3 $(40.2-56.4 \mathrm{~km})$ were both associated with a statistically 
TABLE 1 | The baseline characteristics of the participants by residential distance to the coast.

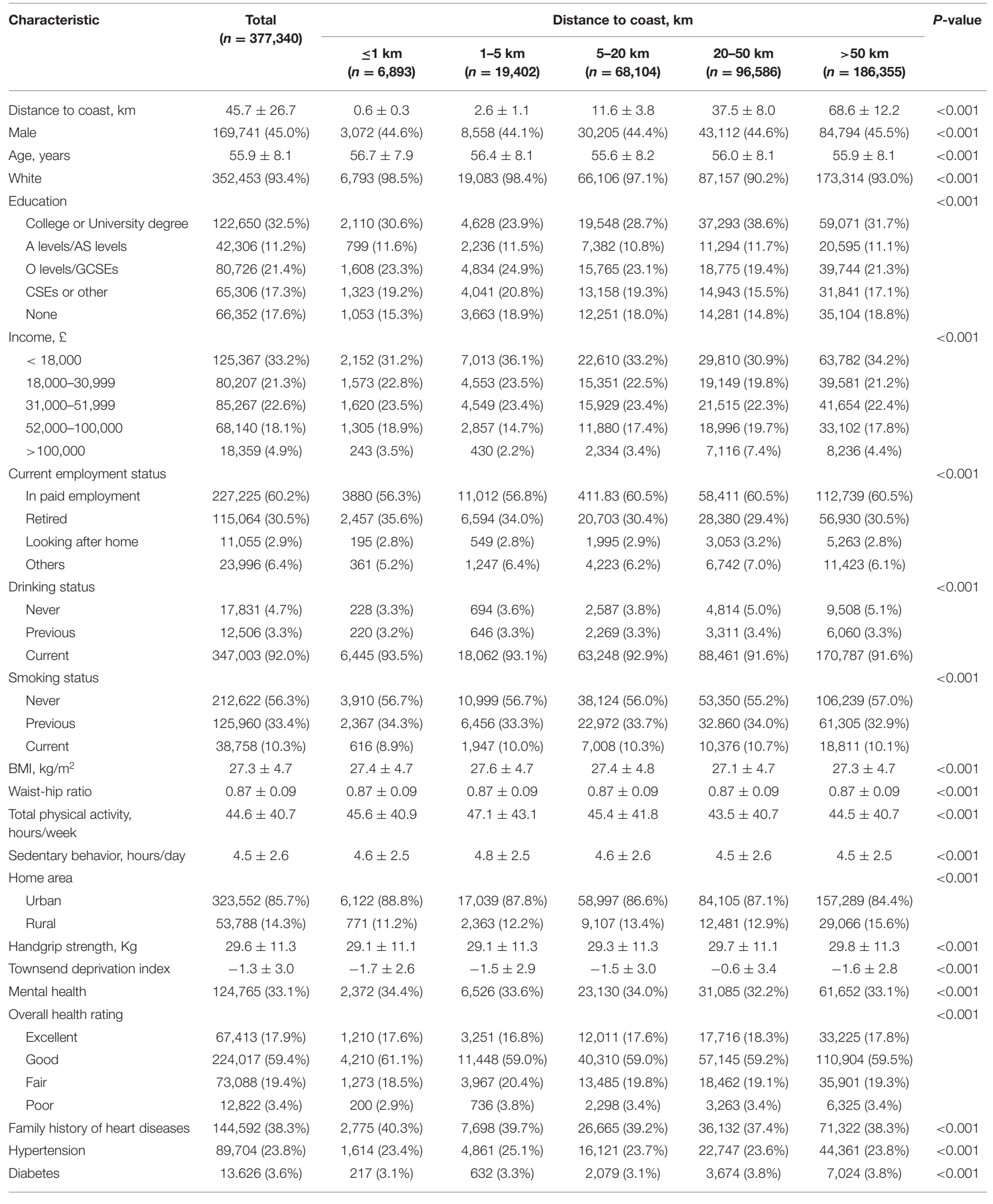


TABLE 1 | Continued

\begin{tabular}{|c|c|c|c|c|c|c|c|}
\hline \multirow[t]{2}{*}{ Characteristic } & \multirow{2}{*}{$\begin{array}{c}\text { Total } \\
(n=377,340)\end{array}$} & \multicolumn{5}{|c|}{ Distance to coast, km } & \multirow[t]{2}{*}{$P$-value } \\
\hline & & $\begin{array}{c}\leq 1 \mathrm{~km} \\
(n=6,893)\end{array}$ & $\begin{array}{c}1-5 \mathrm{~km} \\
(n=19,402)\end{array}$ & $\begin{array}{c}5-20 \mathrm{~km} \\
(n=68,104)\end{array}$ & $\begin{array}{c}20-50 \mathrm{~km} \\
(n=96,586)\end{array}$ & $\begin{array}{c}>50 \mathrm{~km} \\
(n=186,355)\end{array}$ & \\
\hline Aspirin & 36,598 (9.7\%) & 692 (10.0\%) & 1,908 (9.8\%) & $6,431(9.4 \%)$ & $9,426(9.8 \%)$ & $18,141(9.7 \%)$ & 0.132 \\
\hline Anti-hypertension medicine & 20,667 (5.5\%) & $385(5.6 \%)$ & $1,236(6,4 \%)$ & $3,743(5.5 \%)$ & $5,225(5.4 \%)$ & 10,078 (5.4\%) & $<0.001$ \\
\hline Lipid-lowering medicine & $21,138(5.6 \%)$ & $386(5.6 \%)$ & $1,224(6.3 \%)$ & $3.662(5.4 \%)$ & $5,850(6.1 \%)$ & $10,016(5.4 \%)$ & $<0.001$ \\
\hline $\begin{array}{l}\text { Nitrogen dioxide air pollution, } \\
\mathrm{microg} / \mathrm{m}^{3}\end{array}$ & $26.7 \pm 7.6$ & $24.4 \pm 6.0$ & $26.0 \pm 7.0$ & $27.4 \pm 7.3$ & $28.7 \pm 8.9$ & $25.6 \pm 6.8$ & $<0.001$ \\
\hline $\begin{array}{l}\text { Nitrogen oxides, air pollution, } \\
\mathrm{micro} / \mathrm{m}^{3}\end{array}$ & $44.0 \pm 15.5$ & $43.6 \pm 13.8$ & $44.7 \pm 15.5$ & $45.5 \pm 14.8$ & $47.3 \pm 18.2$ & $41.7 \pm 13.8$ & $<0.001$ \\
\hline PM10, microg $/ \mathrm{m}^{3}$ & $16.2 \pm 1.9$ & $15.9 \pm 1.9$ & $16.1 \pm 1.7$ & $16.3 \pm 1.8$ & $16.5 \pm 2.0$ & $16.1 \pm 1.8$ & $<0.001$ \\
\hline $\mathrm{PM} 2.5, \mathrm{microg} / \mathrm{m}^{3}$ & $10.0 \pm 1.0$ & $10.2 \pm 1.1$ & $10.3 \pm 1.2$ & $10.2 \pm 1.1$ & $10.2 \pm 1.1$ & $9.8 \pm 0.9$ & $<0.001$ \\
\hline $\begin{array}{l}\text { Traffic intensity on the nearest } \\
\text { road, vehicles/day }\end{array}$ & $1513.1 \pm 4933.5$ & $934.8 \pm 2339.8$ & $1228.2 \pm 3815.1$ & $1271.9 \pm 4277.8$ & $1764.7 \pm 5606.4$ & $1521.8 \pm 4950.9$ & $<0.001$ \\
\hline $\begin{array}{l}\text { Inverse distance to the nearest } \\
\text { road, } 1 / \text { meters }\end{array}$ & $0.05 \pm 0.07$ & $0.05 \pm 0.07$ & $0.05 \pm 0.07$ & $0.05 \pm 0.07$ & $0.05 \pm 0.07$ & $0.05 \pm 0.08$ & $<0.001$ \\
\hline $\begin{array}{l}\text { Average daytime sound level of } \\
\text { noise pollution, } d B\end{array}$ & $55.4 \pm 4.3$ & $55.3 \pm 3.7$ & $55.3 \pm 4.0$ & $55.2 \pm 4.0$ & $55.9 \pm 4.6$ & $55.2 \pm 4.2$ & $<0.001$ \\
\hline $\begin{array}{l}\text { Average evening sound level of } \\
\text { noise pollution, } \mathrm{dB}\end{array}$ & $51.7 \pm 4.3$ & $51.6 \pm 3.7$ & $51.5 \pm 4.0$ & $51.5 \pm 4.0$ & $52.1 \pm 4.6$ & $51.5 \pm 4.2$ & $<0.001$ \\
\hline $\begin{array}{l}\text { Average night-time sound level } \\
\text { of noise pollution, } \mathrm{dB}\end{array}$ & $46.6 \pm 4.3$ & $46.5 \pm 3.7$ & $46.5 \pm 4.0$ & $46.4 \pm 4.0$ & $47.1 \pm 4.6$ & $46.4 \pm 4.2$ & $<0.001$ \\
\hline Food weight, g & $3263.1 \pm 374.0$ & $3268.4 \pm 388.7$ & $3258.9 \pm 419.2$ & $3264.1 \pm 311.6$ & $3262.8 \pm 444.9$ & $3263.1 \pm 348.3$ & $<0.001$ \\
\hline Energy, KJ & $8826.7 \pm 1284.0$ & $8836.9 \pm 1277.4$ & $8834.5 \pm 1498.0$ & $8835.6 \pm 1065.4$ & $8823.8 \pm 1531.1$ & $8823.8 \pm 1189.4$ & $<0.001$ \\
\hline Protein, g & $81.8 \pm 12.4$ & $81.9 \pm 12.7$ & $81.8 \pm 13.9$ & $81.9 \pm 10.2$ & $81.8 \pm 14.9$ & $81.8 \pm 11.6$ & $<0.001$ \\
\hline Fat, g & $76.8 \pm 14.5$ & $77.0 \pm 15.2$ & $76.9 \pm 16.5$ & $76.8 \pm 12.1$ & $76.8 \pm 17.4$ & $76.7 \pm 13.4$ & $<0.001$ \\
\hline Carbohydrate, g & $258.1 \pm 43.1$ & $258.1 \pm 42.0$ & $258.8 \pm 50.0$ & $258.4 \pm 35.9$ & $257.6 \pm 51.4$ & $258.3 \pm 39.9$ & $<0.001$ \\
\hline Englyst dietary fiber, g & $16.6 \pm 3.1$ & $16.7 \pm 3.4$ & $16.7 \pm 3.5$ & $16.7 \pm 2.6$ & $16.6 \pm 3.7$ & $16.6 \pm 2.9$ & $<0.001$ \\
\hline Sleep duration, hours/day & $7.14 \pm 1.1$ & $7.19 \pm 1.1$ & $7.15 \pm 1.1$ & $7.15 \pm 1.1$ & $7.13 \pm 1.1$ & $7.15 \pm 1.1$ & $<0.001$ \\
\hline
\end{tabular}

TABLE 2 | Association between distance to the coast and incident myocardial infarction (MI).

\begin{tabular}{|c|c|c|c|c|c|c|c|c|}
\hline \multirow[t]{2}{*}{ Subgroup } & \multirow[t]{2}{*}{ Events rate } & \multicolumn{2}{|c|}{ Model 1} & \multicolumn{2}{|c|}{ Model 2} & \multicolumn{3}{|c|}{ Model 3} \\
\hline & & HR (95\% Cl) & $P$-value & HR (95\% Cl) & $P$-value & HR (95\% Cl) & $P$-value & $\begin{array}{l}P \text { for } \\
\text { trend }\end{array}$ \\
\hline Per SD (26.7 km) & 4,059/377,340 & $0.97(0.94,1.00)$ & 0.085 & $0.97(0.94,1.00)$ & 0.092 & $0.98(0.95,1.02)$ & 0.321 & \\
\hline$<1 \mathrm{~km}$ & $94 / 6,893$ & Reference & - & Reference & - & Reference & - & \\
\hline $1-5 \mathrm{~km}$ & $245 / 19,402$ & $0.90(0.71,1.14)$ & 0.372 & $0.89(0.71,1.13)$ & 0.355 & $0.93(0.73,1.18)$ & 0.564 & \\
\hline $5-20 \mathrm{~km}$ & $765 / 68,104$ & $0.82(0.66,1.01)$ & 0.067 & $0.82(0.66,1.01)$ & 0.066 & $0.89(0.71,1.10)$ & 0.280 & \\
\hline $20-50$ km & $906 / 96,586$ & $0.72(0.58,0.89)$ & 0.002 & $0.72(0.58,0.89)$ & 0.002 & $0.79(0.64,0.98)$ & 0.033 & \\
\hline$>50 \mathrm{~km}$ & 2,049/186,355 & $0.77(0.63,0.95)$ & 0.015 & $0.77(0.63,0.95)$ & 0.015 & $0.84(0.68,1.04)$ & 0.109 & \\
\hline Q1 (<14.1km) & $901 / 75,663$ & Reference & - & Reference & - & Reference & - & 0.586 \\
\hline Q2 (14.1-40.2km) & $717 / 75,249$ & $0.84(0.76,0.93)$ & 0.001 & $0.84(0.76,0.93)$ & 0.001 & $0.86(0.78,0.95)$ & 0.003 & \\
\hline Q3 (40.2-56.4 km) & 768/75,723 & $0.82(0.75,0.91)$ & $<0.001$ & $0.82(0.75,0.91)$ & $<0.001$ & $0.85(0.77,0.94)$ & 0.001 & \\
\hline Q4 (56.4-70.2km) & $841 / 75,426$ & $0.90(0.82,0.99)$ & 0.033 & $0.90(0.82,0.99)$ & 0.035 & $0.91(0.83,1.00)$ & 0.058 & \\
\hline Q5 ( $\geq 70.2 \mathrm{~km})$ & $832 / 75,279$ & $0.92(0.84,1.01)$ & 0.079 & $0.92(0.84,1.01)$ & 0.086 & $0.95(0.86,1.04)$ & 0.265 & \\
\hline
\end{tabular}

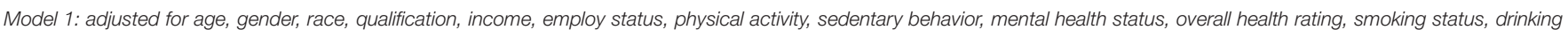

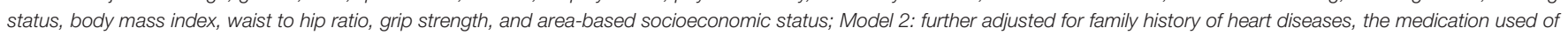

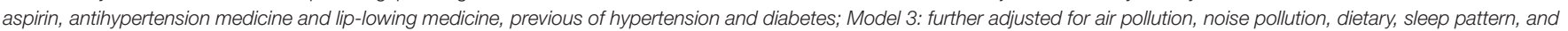
home area population density.

$H R$, hazard ratio; $\mathrm{Cl}$, confident interval; SD, standard difference; $\mathrm{Km}$, kilometer. 


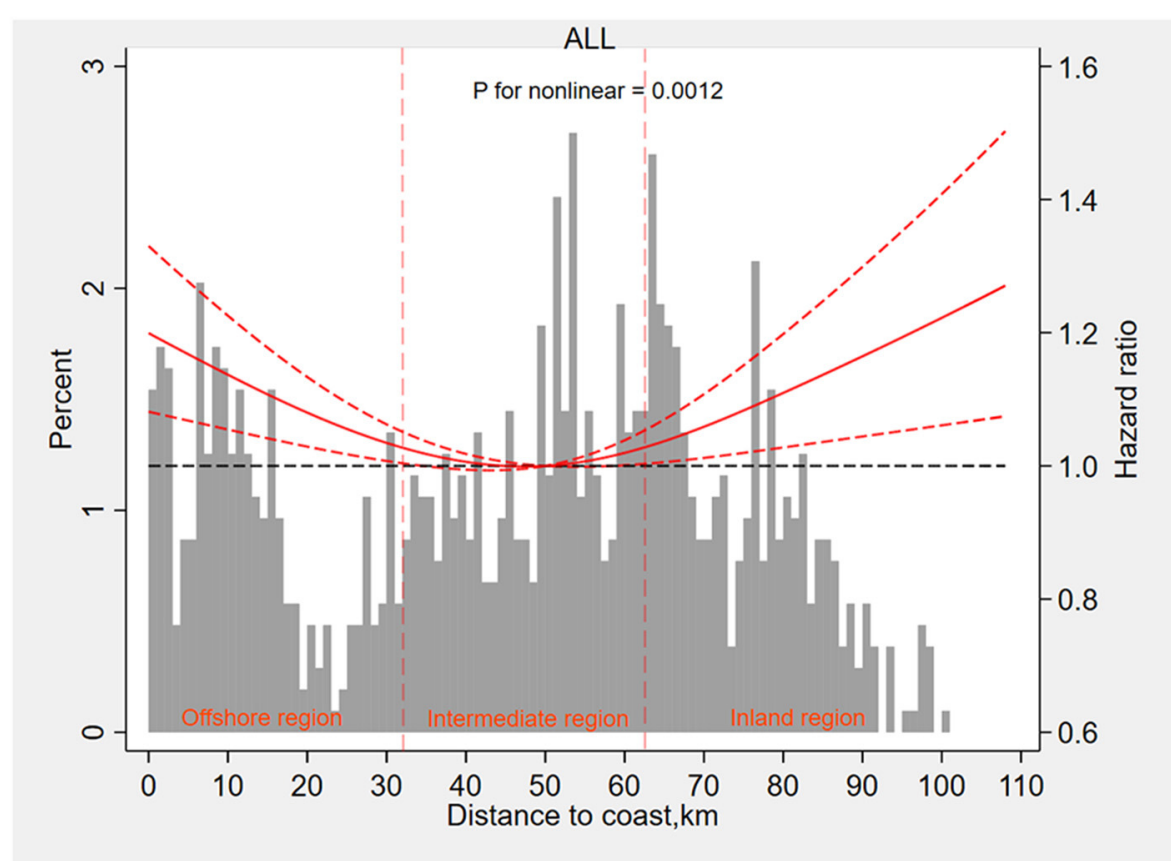

FIGURE 1 | The restricted cubic spline curve to explore potential non-linear patterns between distance to the coast and myocardial infarction. Km, kilometers.

TABLE 3 | Hazard ratio for participants of offshore region and inland region with MI.

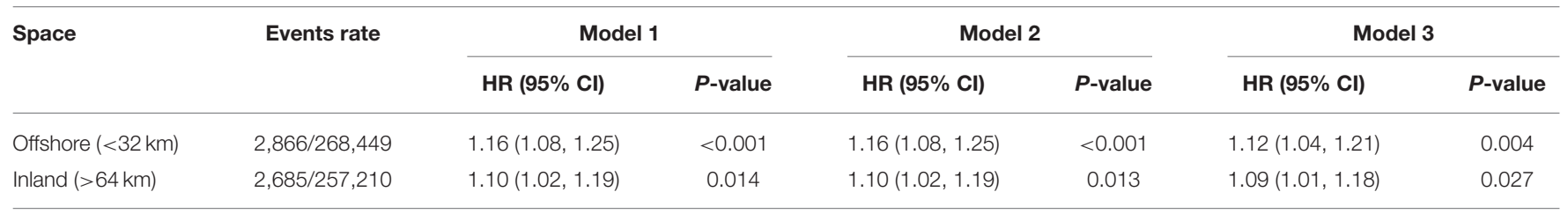

Model 1: adjusted for age, gender, race, qualification, income, employ status, physical activity, sedentary behavior, mental health status, overall health rating, smoking status, drinking status, BMI, waist to hip ratio, grip strength, and area-based socioeconomic status; Model 2: further adjusted for family history of heart diseases, the medication used of aspirin, antihypertension medicine and lip-lowing medicine, previous of hypertension and diabetes; Model 3: further adjusted for air pollution, noise pollution, dietary, sleep pattern, and home area population density.

HR, hazard ratio; $\mathrm{Cl}$, confident interval; $\mathrm{Km}$, kilometer.

significant lower risk of MI (HR 0.86, 95\% CI 0.78-0.95, $p=$ 0.003 ; and $H R \quad 0.85,95 \%$ CI $0.77-0.94, p=0.001$, respectively; $p$ for trend $=0.586$, Table 2 ).

We then applied the restricted cubic spline curve to explore potential non-linear patterns. Figure 1 shows a $U$-shaped relation between distance to coast and MI with a relatively lower risk interval between 32 and $64 \mathrm{~km}$ ( $p$ for non-linear $=0.0012$ ). According to the curve, we divided the population into three categories: offshore region $(<32 \mathrm{~km})$, inland region $(>64 \mathrm{~km})$, and intermediate area $(32-64 \mathrm{~km})$. Using participants of the intermediate region $(32-64 \mathrm{~km})$ as a reference, participants of the offshore region $(<32 \mathrm{~km})$, and inland region $(>64 \mathrm{~km})$ were both associated with a higher risk of incident $\mathrm{MI}$ and HRs were 1.12 (95\% CI 1.04-1.21, $p=0.004$ ) and 1.09 (95\% CI 1.01$1.18, p=0.027)$ after adjusting for all confounders, respectively (Table 3).

Figure 2 shows the associations between participants living in the offshore region $(<32 \mathrm{~km})$ and incident MI in subgroups analyses. $H R$ for the offshore area $(<32 \mathrm{~km})$ was higher in the subgroup with low total physical activity $(<24 \mathrm{~h} /$ week) (HR 1.24, 95\% CI 1.09-1.42, $p=0.001)$, compared with subgroups with moderate and high total physical activity ( $p$ for interaction $=0.043$ ). Figure 3 shows the associations between participants living in the inland region $(>64 \mathrm{~km})$ and incident MI in subgroups stratified by potential effect modifiers. $H R$ for the inland region $(>64 \mathrm{~km})$ was significantly larger in the subgroup of the urban area $(H R 1.12,95 \%$ CI 1.03-1.22, $p=$ 0.007 ), compared with the subgroup of the rural area ( $p$ for interaction 0.065$)$. Also, $H R$ for the inland region $(>64 \mathrm{~km})$ was significantly larger in the subgroup of high $\mathrm{NO}_{2}$ air pollution exposure ( $H R 1.29,95 \% C I 1.11-1.50, p=0.001)$, compared with middle and low $\mathrm{NO}_{2}$ air pollution ( $p$ for interaction $=0.021$ ).

\section{DISCUSSION}

The main finding of the current study was that residential distance to coast had a $U$-shaped relation with incident $\mathrm{MI}$ in 


\begin{tabular}{|c|c|c|c|c|c|c|}
\hline \multicolumn{7}{|c|}{ Offshore } \\
\hline Subgroup & $\begin{array}{c}\text { No. of } \\
\text { Participants }\end{array}$ & $\begin{array}{l}\text { No. of } \\
\text { Events }\end{array}$ & Hazard ratio $(95 \% \mathrm{Cl})$ & $\mathrm{P}$ Value & & $\begin{array}{c}\mathrm{P} \text { for } \\
\text { interaction }\end{array}$ \\
\hline \multicolumn{6}{|l|}{ Sex } & 0.982 \\
\hline Male & 120,492 & 2,042 & $1.12(1.02,1.22)$ & 0.018 & & $10-1$ \\
\hline Female & 147,957 & 824 & $1.12(0.97,1.30)$ & 0.116 & & $\longrightarrow$ \\
\hline \multicolumn{6}{|l|}{ Age, years } & 0.911 \\
\hline$<50$ & 78,176 & 403 & $1.24(1.00,1.52)$ & 0.047 & & $\square$ \\
\hline $50-60$ & 95,956 & 966 & $1.05(0.91,1.19)$ & 0.521 & 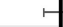 & 10 \\
\hline$\geq 60$ & 94,317 & 1,497 & $1.14(1.02,1.26)$ & 0.020 & & $1-1$ \\
\hline \multicolumn{6}{|l|}{ BMI. $\mathrm{kq} / \mathrm{m}^{2}$} & 0.881 \\
\hline$<24.9$ & 87,759 & 624 & $1.10(0.93,1.30)$ & 0.283 & $H$ & $=1$ \\
\hline 24.9-28.5 & 90,368 & 1,022 & $1.14(1.00,1.30)$ & 0.051 & & $\because$ \\
\hline$\geq 28.5$ & 90,322 & 1,220 & $1.11(0.99,1.26)$ & 0.073 & & -1 \\
\hline Sedentary behaviour, hours/day & & & & & & 0.802 \\
\hline$<3$ & 97,468 & 745 & $1.17(1.00,1.36)$ & 0.045 & & $\square$ \\
\hline $3-6$ & 123,143 & 1,398 & $1.14(1.02,1.27)$ & 0.019 & & $1-1$ \\
\hline$\geq 6$ & 47,838 & 723 & $1.03(0.89,1.21)$ & 0.671 & $\mapsto$ & $\longrightarrow$ \\
\hline Sleep duration, hours/day & & & & & & 0.461 \\
\hline$<7$ & 170,584 & 1,765 & $1.11(1.00,1.22)$ & 0.042 & & -1 \\
\hline $7-8$ & 78,945 & 835 & $1.18(1.02,1.36)$ & 0.027 & & $\longmapsto$ \\
\hline$\geq 8$ & 18,920 & 266 & $1.01(0.78,1.30)$ & 0.957 & $\longmapsto$ & $\longrightarrow$ \\
\hline Total physical activity, hours/week & & & & & & 0.043 \\
\hline$<24$ & 90,639 & 970 & $1.24(1.09,1.42)$ & 0.001 & & $\longmapsto$ \\
\hline $24-44$ & 47,744 & 431 & $0.95(0.78,1.16)$ & 0.620 & $\longmapsto$ & $\longrightarrow$ \\
\hline$\geq 44$ & 130,066 & 1,465 & $1.10(0.98,1.22)$ & 0.095 & & $=-1$ \\
\hline Smoking & & & & & & 0.191 \\
\hline Current & 27,739 & 600 & $1.09(0.92,1.29)$ & 0.326 & $\mapsto$ & $=1$ \\
\hline Former & 89,846 & 1,047 & $1.20(1.06,1.36)$ & 0.005 & & $\longmapsto$ \\
\hline Never & 150,263 & 1,219 & $1.07(0.95,1.20)$ & 0.275 & 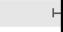 & $=-1$ \\
\hline Drinking & & & & & & 0.710 \\
\hline Current & 246,642 & 2,593 & $1.11(1.02,1.20)$ & 0.014 & & $1=-1$ \\
\hline Former & 9,085 & 128 & $1.27(0.87,1.85)$ & 0.213 & $\mapsto$ & $=$ \\
\hline Never & 12,722 & 145 & $1.29(0.90,1.86)$ & 0.172 & $\mapsto$ & $=$ \\
\hline Income, $£$ & & & & & & 0.577 \\
\hline$<18,000$ & 89,834 & 1,192 & $1.10(0.97,1.24)$ & 0.132 & & $=-1$ \\
\hline $18000-31000$ & 56,661 & 683 & $1.04(0.89,1.22)$ & 0.602 & $\mapsto$ & 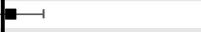 \\
\hline $31000-52000$ & 60,145 & 570 & $1.13(0.95,1.34)$ & 0.175 & 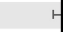 & $=$ \\
\hline$\geq 52000$ & 48,448 & 359 & $1.28(1.03,1.59)$ & 0.028 & & $\longmapsto$ \\
\hline Townsend deprivation index & & & & & & 0.275 \\
\hline$<-3.2$ & 83,168 & 888 & $1.18(1.03,1.36)$ & 0.017 & & $\longmapsto$ \\
\hline$-3.2 \sim-0.66$ & 89,332 & 957 & $1.06(0.92,1.21)$ & 0.429 & $\mapsto$ & $=1$ \\
\hline$\geq-0.66$ & 95,949 & 1,021 & $1.13(0.99,1.29)$ & 0.075 & & $\Rightarrow$ \\
\hline $\begin{array}{l}\text { Seen doctor for nerves, anxiety, tension } \\
\text { or depression }\end{array}$ & & & & & & 0.980 \\
\hline Yes & 88,632 & 954 & $1.13(0.90,1.29)$ & 0.071 & $\mapsto$ & $\Rightarrow$ \\
\hline No & 179,817 & 1,912 & $1.11(1.01,1.23)$ & 0.026 & & -1 \\
\hline Urban & & & & & & 0.229 \\
\hline Yes & 235,054 & 2,500 & $1.14(1.05,1.24)$ & 0.002 & & 1 \\
\hline No & 33,395 & 366 & $1.00(0.81,1.23)$ & 0.973 & $\longmapsto$ & $\longrightarrow$ \\
\hline Nitrogen dioxide air pollution, micro-g/m & & & & & & 0.373 \\
\hline$<23.18$ & 81,345 & 892 & $1.10(0.96,1.26)$ & 0.169 & $\mapsto$ & $=-1$ \\
\hline $23.18-29.47$ & 87,551 & 1,000 & $1.04(0.91,1.18)$ & 0.579 & $\mapsto$ & 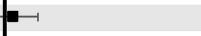 \\
\hline$\geq 29.47$ & 99,553 & 974 & $1.25(1.09,1.43)$ & 0.001 & & $\longmapsto$ \\
\hline Nitrogen oxides air pollution, micro-g/m3 & & & & & & 0.732 \\
\hline$<37.3$ & 80,530 & 870 & $1.18(1.02,1.35)$ & 0.021 & & $\longmapsto$ \\
\hline $37.3-47.33$ & 86,772 & 963 & $1.06(0.93,1.21)$ & 0.417 & $\mapsto$ & $=1$ \\
\hline$\geq 47.33$ & 101,147 & 1,033 & $1.14(1.00,1.30)$ & 0.055 & & $\because$ \\
\hline PM 10, micro-g/m3 & & & & & & 0.636 \\
\hline$<15.60$ & 87,298 & 1,003 & $1.09(0.95,1.23)$ & 0.218 & & $t=1$ \\
\hline $15.60-16.57$ & 86,197 & 946 & $1.13(0.99,1.29)$ & 0.077 & & $\because$ \\
\hline$\geq 16.57$ & 94,954 & 917 & $1.16(1.01,1.34)$ & 0.039 & & $\square$ \\
\hline PM 2.5, micro-g/m3 & & & & & & 0.839 \\
\hline$<9.54$ & 79,545 & 843 & $1.14(0.99,1.31)$ & 0.069 & & $\because$ \\
\hline $9.54-10.31$ & 86,098 & 901 & $1.10(0.96,1.26)$ & 0.165 & & $=1$ \\
\hline$\geq 10.31$ & 102,806 & 1,122 & $1.12(0.99,1.27)$ & 0.073 & & -1 \\
\hline Noise pollution at daytime, $\mathrm{dB}$ & & & & & & 0.813 \\
\hline$<53.38$ & 86,913 & 923 & $1.11(0.97,1.28)$ & 0.120 & & -1 \\
\hline $53.38-55.40$ & 86,114 & 883 & $1.12(0.97,1.29)$ & 0.110 & & $=1$ \\
\hline$\geq 55.40$ & 95,422 & 1,060 & $1.13(1.00,1.29)$ & 0.053 & & $=-1$ \\
\hline Hypertension & & & & & & 0.813 \\
\hline Yes & 64,097 & 1,051 & $1.13(0.99,1.28)$ & 0.072 & & $=-1$ \\
\hline No & 204,352 & 1,815 & $1.12(1.02,1.23)$ & 0.022 & & $1=-1$ \\
\hline $\begin{array}{l}\text { Excluding participants with Ml occurring } \\
\text { within the first } 24 \text { months }\end{array}$ & 267,908 & 2,325 & $1.12(1.03,1.22)$ & 0.011 & & $1-1$ \\
\hline & & & & 0. & 0.8 & $\begin{array}{llll}1 & 1.2 & 1.4 & 1.6\end{array}$ \\
\hline
\end{tabular}

FIGURE 2 | The associations between participants living in the offshore region ( $<32 \mathrm{~km})$ and incident myocardial infarction (MI) in subgroups analyses. BMI, body mass index; PM, particulate matter. 


\begin{tabular}{|c|c|c|c|c|c|c|c|}
\hline \multicolumn{8}{|c|}{ Inland } \\
\hline Subgroup & $\begin{array}{c}\text { No. of } \\
\text { Participants }\end{array}$ & $\begin{array}{l}\text { No. of } \\
\text { Events }\end{array}$ & Hazard ratio $(95 \% \mathrm{Cl})$ & $P$ Value & & & $\begin{array}{c}\mathrm{P} \text { for } \\
\text { interaction }\end{array}$ \\
\hline Sex & & & & & & 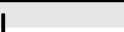 & 0.162 \\
\hline Male & 116,278 & 1,955 & $1.13(1.03,1.24)$ & 0.009 & & $1-1$ & \\
\hline Female & 140,932 & 730 & $1.02(0.88,1.19)$ & 0.803 & $\mapsto$ & 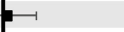 & \\
\hline Age, years & & & & & & & 0.205 \\
\hline$<50$ & 73,902 & 350 & $1.03(0.82,1.29)$ & 0.800 & $\longmapsto$ & $\longrightarrow$ & \\
\hline $50-60$ & 92,792 & 957 & $1.19(1.04,1.36)$ & 0.009 & & $\longmapsto$ & \\
\hline$\geq 60$ & 90,516 & 1,378 & $1.04(0.94,1.17)$ & 0.449 & $H$ & -1 & \\
\hline BMI. $\mathrm{kq} / \mathrm{m}^{2}$ & & & & & & & 1.000 \\
\hline$<24.9$ & 86,075 & 608 & $1.11(0.94,1.32)$ & 0.216 & t & $=1$ & \\
\hline 24.9-28.5 & 86,660 & 958 & $1.10(0.96,1.25)$ & 0.167 & t & $\Rightarrow$ & \\
\hline$\geq 28.5$ & 84,475 & 1,119 & $1.09(0.96,1.23)$ & 0.177 & t & $t=1$ & \\
\hline Sedentary behaviour, hours/day & & & & & & & 0.830 \\
\hline$<3$ & 96,434 & 721 & $1.08(0.92,1.25)$ & 0.358 & $H$ & $=-1$ & \\
\hline $3-6$ & 116,866 & 1,319 & $1.11(0.99,1.24)$ & 0.065 & & -1 & \\
\hline$\geq 6$ & 43,910 & 645 & $1.08(0.92,1.27)$ & 0.373 & $H$ & \pm & \\
\hline Sleep duration, hours/day & & & & & & & 0.592 \\
\hline$<7$ & 163,685 & 1,657 & $1.12(1.01,1.23)$ & 0.034 & & $=-1$ & \\
\hline 7-8 & 75,817 & 783 & $1.10(0.95,1.27)$ & 0.199 & & 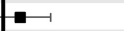 & \\
\hline$\geq 8$ & 17,708 & 245 & $0.96(0.73,1.25)$ & 0.736 & $\longmapsto$ & $\longrightarrow$ & \\
\hline Total physical activity, hours/week & & & & & & & 0.247 \\
\hline$<24$ & 87,886 & 899 & $1.19(1.04,1.37)$ & 0.011 & & $\longmapsto$ & \\
\hline $24-44$ & 45,998 & 414 & $0.98(0.80,1.20)$ & 0.815 & $\longmapsto$ & $\longrightarrow$ & \\
\hline$\geq 44$ & 123,326 & 1,372 & $1.08(0.96,1.20)$ & 0.196 & & $=-1$ & \\
\hline Smoking & & & & & & & 0.252 \\
\hline Current & 26,761 & 558 & $1.16(0.97,1.39)$ & 0.095 & & $\because$ & \\
\hline Former & 85,401 & 974 & $1.14(1.00,1.30)$ & 0.044 & & -1 & \\
\hline Never & 145,048 & 1,153 & $1.03(0.92,1.17)$ & 0.589 & $H$ & 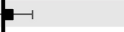 & \\
\hline Drinking & & & & & & & 0.169 \\
\hline Current & 235,433 & 2,407 & $1.06(0.98,1.16)$ & 0.149 & & $e_{-1}$ & \\
\hline Former & 8,526 & 132 & $1.59(1.10,2.31)$ & 0.014 & & $\longmapsto$ & \\
\hline Never & 13,251 & 146 & $1.32(0.93,1.86)$ & 0.118 & $H$ & 10 & \\
\hline Income, $£$ & & & & & & & 0.709 \\
\hline$<18,000$ & 85,788 & 1,104 & $1.07(0.95,1.21)$ & 0.285 & 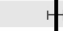 & $=-1$ & \\
\hline $18000-31000$ & 53,114 & 638 & $1.08(0.92,1.27)$ & 0.349 & $H$ & $=-1$ & \\
\hline $31000-52000$ & 57,051 & 537 & $1.06(0.89,1.26)$ & 0.534 & $\mapsto$ & $=$ & \\
\hline$\geq 52000$ & 47,200 & 337 & $1.19(0.95,1.48)$ & 0.139 & t & $\square$ & \\
\hline Townsend deprivation index & & & & & & & 0.155 \\
\hline$<-3.2$ & 81,339 & 825 & $1.07(0.93,1.24)$ & 0.315 & $H$ & $=1$ & \\
\hline$-3.2 \sim-0.66$ & 82,244 & 932 & $1.03(0.90,1.18)$ & 0.639 & $\mapsto$ & -1 & \\
\hline$\geq-0.66$ & 88,567 & 928 & $1.19(1.04,1.37)$ & 0.014 & & $\longmapsto$ & \\
\hline $\begin{array}{l}\text { Seen doctor for nerves, anxiety, tension } \\
\text { or depression }\end{array}$ & & & & & & & 0.642 \\
\hline Yes & 84,183 & 892 & $1.12(0.98,1.29)$ & 0.103 & & -1 & \\
\hline No & 173,027 & 1,793 & $1.08(0.98,1.19)$ & 0.117 & & $=-1$ & \\
\hline Urban & & & & & & & 0.065 \\
\hline Yes & 221,755 & 2,309 & $1.12(1.03,1.22)$ & 0.007 & & $1-1$ & \\
\hline No & 35,455 & 376 & $0.96(0.78,1.18)$ & 0.661 & $\longmapsto$ & $\longrightarrow$ & \\
\hline Nitrogen dioxide air pollution, micro- $\mathrm{g} / \mathrm{m}$ & & & & & & & 0.021 \\
\hline$<23.18$ & 84,605 & 942 & $1.11(0.98,1.27)$ & 0.106 & & -1 & \\
\hline $23.18-29.47$ & 85,066 & 903 & $0.97(0.85,1.11)$ & 0.644 & $\mapsto$ & -1 & \\
\hline$\geq 29.47$ & 87,539 & 840 & $1.29(1.11,1.50)$ & 0.001 & & $\longmapsto$ & \\
\hline Nitrogen oxides air pollution, micro-g/m3 & & & & & & & 0.200 \\
\hline$<37.3$ & 87,348 & 943 & $1.13(1.00,1.29)$ & 0.058 & & 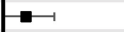 & \\
\hline $37.3-47.33$ & 86,539 & 911 & $1.00(0.87,1.14)$ & 0.969 & $\mapsto$ & 1 & \\
\hline$\geq 47.33$ & 83,323 & 831 & $1.18(1.01,1.37)$ & 0.033 & & 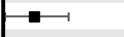 & \\
\hline PM 10, micro-g/m3 & & & & & & & 0.774 \\
\hline$<15.60$ & 83,896 & 954 & $1.06(0.93,1.21)$ & 0.359 & t & $=-1$ & \\
\hline $15.60-16.57$ & 84,265 & 902 & $1.11(0.97,1.27)$ & 0.144 & & 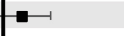 & \\
\hline$\geq 16.57$ & 89,049 & 829 & $1.13(0.97,1.31)$ & 0.113 & & $=1$ & \\
\hline PM 2.5, micro-g/m3 & & & & & & & 0.447 \\
\hline$<9.54$ & 89,860 & 955 & $1.13(0.88,1.03)$ & 0.059 & $\mapsto$ & a & \\
\hline $9.54-10.31$ & 88,211 & 898 & $1.02(0.89,1.16)$ & 0.830 & $\mapsto$ & -1 & \\
\hline$\geq 10.31$ & 79,139 & 832 & $1.14(0.98,1.32)$ & 0.098 & & 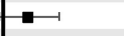 & \\
\hline Noise pollution at daytime, $\mathrm{dB}$ & & & & & & & 0.142 \\
\hline$<53.38$ & 86,025 & 935 & $1.18(1.03,1.34)$ & 0.016 & & $\longmapsto$ & \\
\hline $53.38-55.40$ & 82,893 & 841 & $1.11(0.97,1.28)$ & 0.134 & & 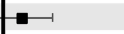 & \\
\hline$\geq 55.40$ & 88,292 & 909 & $0.99(0.86,1.13)$ & 0.837 & $\mapsto$ & -1 & \\
\hline Hypertension & & & & & & & 0.445 \\
\hline Yes & 61,090 & 990 & $1.12(0.99,1.28)$ & 0.080 & & -1 & \\
\hline No & 196,120 & 1,695 & $1.08(0.98,1.20)$ & 0.124 & & $=-1$ & \\
\hline $\begin{array}{l}\text { Excluding participants with MI occurring } \\
\text { within the first } 24 \text { months }\end{array}$ & 256,710 & 2,185 & $1.09(1.00,1.19)$ & 0.048 & & -1 & \\
\hline & & & & 0.6 & 0.8 & $\begin{array}{llll}1 & 1.2 & 1.4 & 1.6\end{array}$ & \\
\hline
\end{tabular}

FIGURE 3 | The associations between participants living in the inland region (>64 km) and incident Ml in subgroups analyses. BMI, body mass index; PM, particulate matter. 


\section{Distance to coast and incident myocardial infarction}

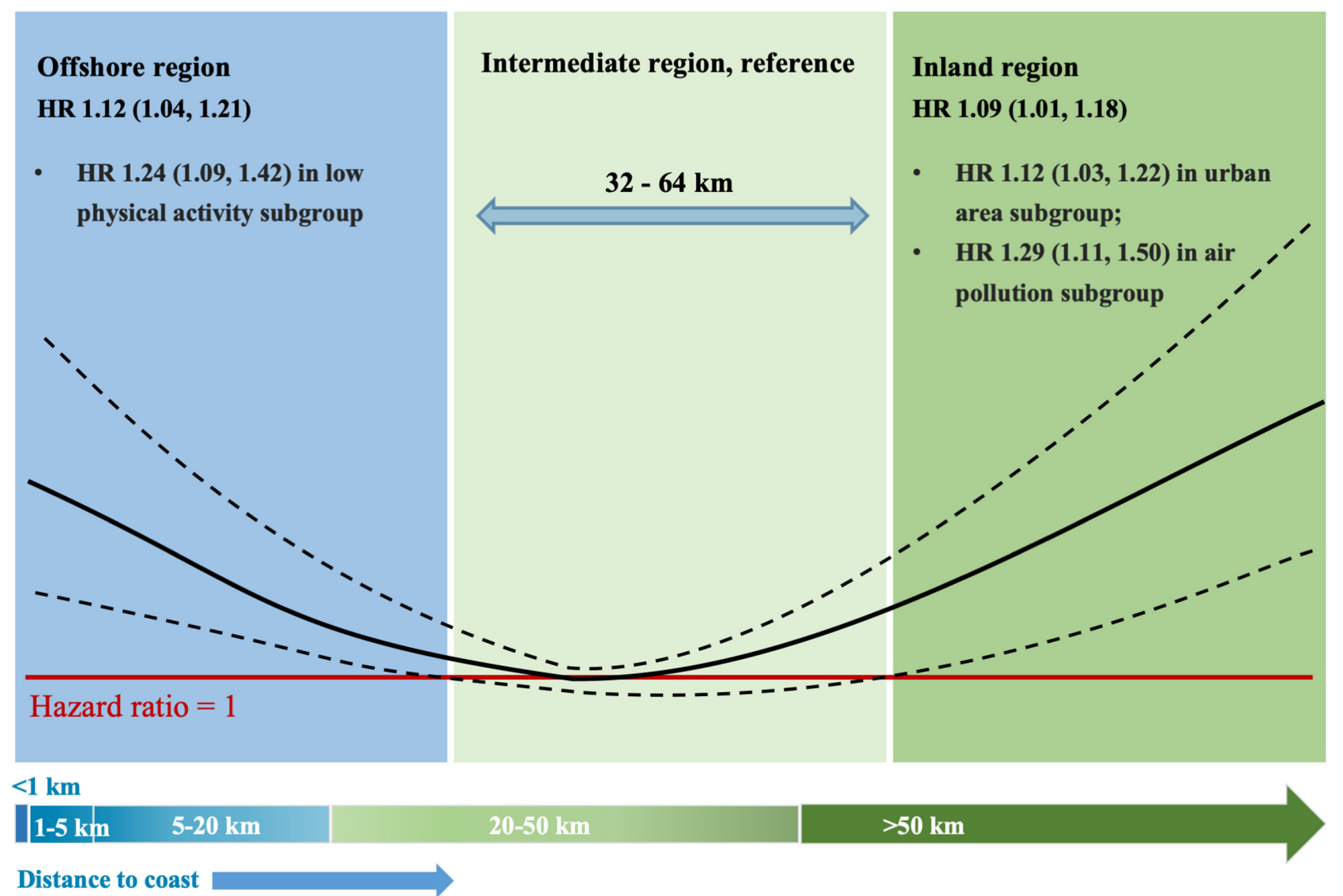

FIGURE 4 | The non-linear pattern between distance to the coast and MI. HR, hazard ratio.

over 370,000 individuals followed for over 3.0 million personyears and that both residents of the offshore region $(<32 \mathrm{~km})$ and that of the inland region $(>64 \mathrm{~km}$ ) had around $10 \%$ increase risk of MI compared with residents of the intermediate region (32$64 \mathrm{~km}$ ) (Figure 4). Moreover, the associations of offshore region and inland region with incident MI were modified by various factors. Participants of offshore regions with low total physical activity had a higher risk of MI (increased by $24 \%$ ), suggesting that this subgroup may benefit from increased physical activity. Meanwhile, participants of inland regions living in the urban area or exposed to high $\mathrm{NO}_{2}$ air pollution had a higher risk of $\mathrm{MI}$ (increased by 12 and 29\%, respectively), suggesting that urban environment improvement and air pollution control played a crucial role in these population. The results were mostly consistent in a series of sensitivity and subgroup analyses.

Unlike previous research that commonly reported linear associations, we investigated the non-linear association of coastal proximity with disease outcome. Our findings highlight the complex and diverse associations between residential distance to the coast and incident MI. Although modifying the residential environment tends to be problematic in the short term, our findings suggest that targeting based on coastal proximity and various effect modifiers (physical activity, population density, and air pollution) could help identify highrisk individuals and provide personalized interventions for diverse populations. However, the classification of the offshore, inland, and intermediate regions was constructed for illustrative purposes; hence the cut-off values of distance to coast identified in this study cannot be simply generalized. This classification is more about raising public awareness of the residential location than about being implemented as a real-world tool. The harmful effect of residential distance to coast on incident MI may vary with the coastline's distance and is regulated by various factors. Therefore, when possible, advice on the living environment and health should be personalized.

Scientific and public interest in the role of the natural outdoor environment in preventing CVDs is growing. A tremendous body of studies has provided evidence on the associations between various outdoor environment attributes, behavioral pathways, CV risk factors, and mortality (18-21). A metaanalysis of longitudinal studies found inverse relationships between neighborhood walkability and risk factors, such as 
obesity, hypertension, and type 2 diabetes mellitus (20). Similarly, another meta-analysis found that more residential greenspace was associated with reduced CVD mortality (22). The links between air and noise pollution, stress, and CVDs have also been recognized (23). The previous research suggested that living near and regularly visiting the coast or other large waterbodies was associated with better general and mental health. However, most of the outcomes used were self-reported and not diseasespecific (24-26). However, the association of residential distance to the coast with CVD incidence is relatively less well studied, and high-quality epidemiological evidence was too scant to draw a conclusion. To investigate this issue at a broad level of disease specificity, we set out to address the question: does the incidence of MI increase or decrease with proximity to the coast?

The results of the study were somewhat unexpected, particularly the finding that MI risk increased in coastal areas, which contradicts most of the previous studies that have shown the benefits of living near the sea. However, our study was based on sufficient confounding adjustment and subgroup analyses, and the results were reliable and consistent. One possible explanation is that the same environment attribute might have diverse effects on cardiometabolic risk factors and different population subgroups. For example, whereas high population density might help improve the weight status through better availability of physical activity destinations and healthy food options, high population density might also have adverse effects on airway and CVD through more exposure to air pollution. Similarly, a previous study showed that individuals living in the $<1 \mathrm{~km}$ coastal category had an average a $4 \mathrm{nmol} / \mathrm{l}$ higher vitamin $\mathrm{D}$ status compared to those living inland through increased solar irradiance, which can provide benefits in terms of vitamin D status but may also pose a risk due to higher skin cancer rates (27). Examining single environmental attributes with multiple behaviors and risk factors in single studies can provide insights into the differential effects of the natural environment on CVD. Further interdisciplinary research initiatives involving cardiology and urban design researchers must disentangle the complex relationships between the residential coastal distance and CVD.

Strengths of this study include its prospective design, a large sample size with harmonized exposure, health, and covariate data. We could adjust for a wide range of health, demographic, behavioral, and environmental confounders. The possibility of confounding was dealt with through statistical adjustment for a wide range of covariates, such as health, demographic, behavioral, and ecological confounders, and a series of sensitivity analyses. This study has several limitations. First, we were not able to consider the impact on the exposure of residential changes during follow-up, which will contribute to misclassification of long-term exposure relevant to the development of MI. These misclassifications are believed to be non-differential for cases, and non-cases likely bias the risk estimates toward the null. Second, as is the case for any observational study, residual confounding is always possible, and associations may not imply causation. We cannot rule out the possibility of residual confounding by other unaccounted factors, such as coastal climate, humidity, and sun exposure. Third, the UK Biobank represents the general population for age, sex, ethnicity, and deprivation within the age range recruited but is not representative in other regards, which may indicate a healthy volunteer selection bias. While this limits the ability to generalize prevalence rates, it should be possible to generalize the estimates of the associations' magnitude. Forth, the offshore, inland, and intermediate regions were constructed for illustrative purposes rather than as a tool ready for implementation. The cut-off values of distance to coast $(32 \mathrm{~km}, 64 \mathrm{~km})$ have not been validated.

\section{CONCLUSIONS}

The study found a $U$-shaped association between residential distance to the coast and incident MI. Moreover, the association of offshore region with incident MI was modified by total physical activity. The association of inland region with incident MI was limited by urban/rural area or $\mathrm{NO}_{2}$ air pollution. Our findings highlight the complex and diverse associations between residential distance to the coast and incident MI, and residential advice should be personalized.

\section{DATA AVAILABILITY STATEMENT}

The datasets presented in this study can be found in online repositories. The names of the repository/repositories and accession number(s) can be found in the article/Supplementary Material.

\section{AUTHOR CONTRIBUTIONS}

ZX-d performed statistical analysis. LL-Z handled funding and supervision. LX-X acquired the data. ZS- $z$ conceived and designed the research. HX drafted the manuscript and made critical revision of the manuscript for critical intellectual content. All authors contributed to the article and approved the submitted version.

\section{FUNDING}

The UK Biobank was supported by the Welcome Trust, Medical Research Council, Department of Health, Scottish Government, and the Northwest Regional Development Agency. It has also had funding from the Welsh Assembly Government and the British Heart Foundation. The research was designed, conducted, analyzed, and interpreted by the authors entirely independently of the funding sources.

\section{SUPPLEMENTARY MATERIAL}

The Supplementary Material for this article can be found online at: https://www.frontiersin.org/articles/10.3389/fcvm. 2022.752964/full\#supplementary-material 


\section{REFERENCES}

1. Koohsari MJ, McCormack GR, Nakaya T, Oka K. Neighbourhood built environment and cardiovascular disease: knowledge and future directions. Nat Rev Cardiol. (2020) 17:261-3. doi: 10.1038/s41569-020-0343-6

2. Gascon M, Sánchez-Benavides G, Dadvand P, Martínez D, Gramunt N, Gotsens X, et al. Long-term exposure to residential green and blue spaces and anxiety and depression in adults: a cross-sectional study. Environ Res. (2018) 162:231-9. doi: 10.1016/j.envres.2018.01.012

3. Huang B, Liu Y, Feng Z, Pearce JR, Wang R, Zhang Y, et al. Residential exposure to natural outdoor environments and general health among older adults in Shanghai, China. Int J Equity Health. (2019) 18:178. doi: 10.1186/s12939-019-1081-4

4. Pasanen TP, White MP, Wheeler BW, Garrett JK, Elliott LR. Neighbourhood blue space, health and wellbeing: the mediating role of different types of physical activity. Environ Int. (2019) 131:105016. doi: 10.1016/j.envint.2019.105016

5. Wood SL, Demougin PR, Higgins S, Husk K, Wheeler BW, White M. Exploring the relationship between childhood obesity and proximity to the coast: a rural/urban perspective. Health Place. (2016) 40:129-36. doi: 10.1016/j.healthplace.2016.05.010

6. Garrett JK, Clitherow TJ, White MP, Wheeler BW, Fleming LE. Coastal proximity and mental health among urban adults in England: The moderating effect of household income. Health Place. (2019) 59:102200. doi: 10.1016/j.healthplace.2019.102200

7. Collins R. What makes UK Biobank special? Lancet. Mar. (2012) 379:1173-4. doi: 10.1016/S0140-6736(12)60404-8

8. Sudlow C, Gallacher J, Allen N, Beral V, Burton P, Danesh J, et al. UK biobank: an open access resource for identifying the causes of a wide range of complex diseases of middle and old age. PLoS Med. (2015) 12:e1001779. doi: 10.1371/journal.pmed.1001779

9. Alcock I, White MP, Wheeler BW, Fleming LE, Depledge MH. Longitudinal effects on mental health of moving to greener and less green urban areas. Environ Sci Technol. (2014) 48:1247-55. doi: 10.1021/es403688w

10. White P, Ruble CL, Lane ME. The effect of changes in land use on nitrate concentration in water supply wells in southern Chester County, Pennsylvania. Environ Monit Assess. (2013) 185:643-51. doi: 10.1007/s10661-012-2581-5

11. Wheeler BW, White M, Stahl-Timmins W, Depledge MH. Does living by the coast improve health and wellbeing? Health Place. (2012) 18:1198-201. doi: 10.1016/j.healthplace.2012.06.015

12. Aveyard P, Manaseki S, Chambers J. The relationship between mean birth weight and poverty using the townsend deprivation score and the super profile classification system. Public Health. (2002) 116:308-14. doi: 10.1016/S0033-3506(02)00554-1

13. Liu B, Young H, Crowe FL, Benson VS, Spencer EA, Key TJ, et al. Development and evaluation of the Oxford WebQ, a low-cost, webbased method for assessment of previous $24 \mathrm{~h}$ dietary intakes in large-scale prospective studies. Public Health Nutr. (2011) 14:1998-2005. doi: $10.1017 /$ S1368980011000942

14. Guo W, Bradbury KE, Reeves GK, Key TJ. Physical activity in relation to body size and composition in women in UK Biobank. Ann Epidemiol. (2015) 25:406-13.e6. doi: 10.1016/j.annepidem.2015.01.015

15. Celis-Morales CA, Petermann F, Hui L, Lyall DM, Iliodromiti S, McLaren J, et al. Associations between diabetes and both cardiovascular disease and allcause mortality are modified by grip strength: evidence from UK Biobank, a prospective population-based cohort study. Diabetes Care. (2017) 40:1710-8. doi: 10.2337/dc17-0921

16. Doiron D, de Hoogh K, Probst-Hensch N, Fortier I, Cai Y, De Matteis $\mathrm{S}$, et al. Air pollution, lung function and COPD: results from the population-based UK Biobank study. Eur Respir J. (2019). 54:1802140. doi: 10.1183/13993003.02140-2018

17. Kephalopoulos S, Paviotti M, Anfosso-Lédée F, Van Maercke D, Shilton S, Jones N. Advances in the development of common noise assessment methods in Europe: the CNOSSOS-EU framework for strategic environmental noise mapping. Sci Total Environ. (2014) 482-3:400-10. doi: 10.1016/j.scitotenv.2014.02.031

18. Nieuwenhuijsen MJ. Influence of urban and transport planning and the city environment on cardiovascular disease. Nat Rev Cardiol. (2018) 15:432-8. doi: 10.1038/s41569-018-0003-2

19. Stevenson M, Thompson J, de Sá TH, Ewing R, Mohan D, McClure $\mathrm{R}$, et al. Land use, transport, and population health: estimating the health benefits of compact cities. Lancet. (2016) 388:2925-35. doi: 10.1016/S0140-6736(16)30067-8

20. Chandrabose M, Rachele JN, Gunn L, Kavanagh A, Owen N, Turrell G, et al. Built environment and cardio-metabolic health: systematic review and meta-analysis of longitudinal studies. Obes Rev. (2019) 20:41-54. doi: 10.1111/obr.12759

21. Garg PK, Jorgensen N, Moore K, Soliman EZ, Heckbert SR. Neighborhood environments and risk of incident atrial fibrillation: the multiethnic study of atherosclerosis. Eur J Prev Cardiol. (2020) 27:1440-1. doi: $10.1177 / 2047487319885196$

22. Gascon M, Triguero-Mas M, Martínez D, Dadvand P, Rojas-Rueda D, Plasència $A$, et al. Residential green spaces and mortality: a systematic review. Environ Int. (2016) 86:60-7. doi: 10.1016/j.envint.2015.10.013

23. Al-Kindi SG, Brook RD, Biswal S, Rajagopalan S. Environmental determinants of cardiovascular disease: lessons learned from air pollution. Nat Rev Cardiol. (2020) 17:656-72. doi: 10.1038/s41569-020-0371-2

24. Garrett JK, White MP, Huang J, Ng S, Hui Z, Leung C, et al. Urban blue space and health and wellbeing in Hong Kong: results from a survey of older adults. Health Place. (2019) 55:100-10. doi: 10.1016/j.healthplace.2018.11.003

25. Dempsey S, Devine MT, Gillespie T, Lyons S, Nolan A. Coastal blue space and depression in older adults. Health Place. (2018) 54:110-7. doi: 10.1016/j.healthplace.2018.09.002

26. Dzhambov AM, Markevych I, Hartig T, Tilov B, Arabadzhiev Z, Stoyanov D, et al. Multiple pathways link urban green- and bluespace to mental health in young adults. Environ Res. (2018) 166:223-3. doi: 10.1016/j.envres.2018.06.004

27. Cherrie MP, Wheeler BW, White MP, Sarran CE, Osborne NJ. The coastal climate is associated with elevated solar irradiance and higher 25(OH)D level. Environ Int. (2015) 77:76-84. doi: 10.1016/j.envint.2015.01.005

Conflict of Interest: The authors declare that the research was conducted in the absence of any commercial or financial relationships that could be construed as a potential conflict of interest.

Publisher's Note: All claims expressed in this article are solely those of the authors and do not necessarily represent those of their affiliated organizations, or those of the publisher, the editors and the reviewers. Any product that may be evaluated in this article, or claim that may be made by its manufacturer, is not guaranteed or endorsed by the publisher.

Copyright (c) 2022 Xiao-dong, Shao-zhao, Xun, Xin-xue and Li-zhen. This is an open-access article distributed under the terms of the Creative Commons Attribution License (CC BY). The use, distribution or reproduction in other forums is permitted, provided the original author(s) and the copyright owner(s) are credited and that the original publication in this journal is cited, in accordance with accepted academic practice. No use, distribution or reproduction is permitted which does not comply with these terms. 